University of Wollongong

Research Online

Faculty of Engineering and Information

Faculty of Engineering and Information

Sciences - Papers: Part B

Sciences

2020

Broken lines path following algorithm for a water-jet propulsion USV with disturbance uncertainties

Yujiao Zhao

Xin Qi

Atilla Incecik

Yong Ma

Zhixiong Li

University of Wollongong, lizhixio@uow.edu.au

Follow this and additional works at: https://ro.uow.edu.au/eispapers1

Part of the Engineering Commons, and the Science and Technology Studies Commons

Research Online is the open access institutional repository for the University of Wollongong. For further information contact the UOW Library: research-pubs@uow.edu.au 


\title{
Broken lines path following algorithm for a water-jet propulsion USV with disturbance uncertainties
}

\author{
Abstract \\ 2020 Elsevier Ltd To fulfill autonomous navigation of unmanned surface vehicle, it is pivotal to resolve \\ unmanned surface vehicle path following issue. However, the problem that we are concerned with is \\ hindered by unmanned surface vehicle's nonlinearity and the disturbance uncertainties brought by the \\ navigation environment. In this paper, we focus on path following problem for a jet driven underactuated \\ unmanned surface vehicle. The stressed followed paths in practical application are broken lines \\ connected by continuous straight lines rather than smooth lines. By calculating the virtual target on each \\ straight line in following the unmanned surface vehicle dynamically, we propose a broken lines path \\ following algorithm for the unmanned surface vehicle in an uncertain environment. By employing our \\ unmanned surface vehicle experiment platform that we developed a series of numerical simulations and \\ physical tests have been carried out. Results verify the superiority and effectiveness of our broken lines \\ path following algorithm for unmanned surface vehicle especially in continuous broken lines following \\ scenarios.

\section{Disciplines} \\ Engineering | Science and Technology Studies

\section{Publication Details} \\ Zhao, Y., Qi, X., Incecik, A., Ma, Y. \& Li, Z. (2020). Broken lines path following algorithm for a water-jet \\ propulsion USV with disturbance uncertainties. Ocean Engineering, 201
}




\title{
Broken Lines Path Following Algorithm for a Water-Jet Propulsion USV with Disturbance Uncertainties
}

\author{
Yujiao Zhao ${ }^{\mathrm{a}, \mathrm{b}}$, Xin $\mathrm{Qi}^{\mathrm{b}}$, Atilla Incecik ${ }^{\mathrm{c}}$, Yong $\mathrm{Ma}^{\mathrm{a}, \mathrm{e}, *}$, Zhixiong $\mathrm{Li}^{\mathrm{d}, *}$ \\ ${ }^{a}$ School of Navigation, Wuhan University of Technology, Wuhan, Hubei, 430063, China \\ ${ }^{b}$ School of Computer Science and Technology, Wuhan University of Technology, Wuhan, Hubei, 430063, China \\ ${ }^{c}$ Department of Naval Architecture and Marine Engineering, University of Strathclyde, Glasgow, G11XQ, UK. \\ ${ }^{d}$ School of Mechanical, Materials, Mechatronic and Biomedical Engineering, University of Wollongong, Wollongong, NSW 2522, Australia. \\ ${ }^{e}$ Hubei Key Laboratory of Inland Shipping Technology, Wuhan, Hubei, 430063, China
}

\begin{abstract}
To fulfill autonomous navigation of unmanned surface vehicle, it is pivotal to resolve unmanned surface vehicle path following issue. However, the problem that we are concerned with is hindered by unmanned surface vehicle's nonlinearity and the disturbance uncertainties brought by the navigation environment. In this paper, we focus on path following problem for a jet driven underactuated unmanned surface vehicle. The stressed followed paths in practical application are broken lines connected by continuous straight lines rather than smooth lines. By calculating the virtual target on each straight line in following the unmanned surface vehicle dynamically, we propose a broken lines path following algorithm for the unmanned surface vehicle in an uncertain environment. By employing our unmanned surface vehicle experiment platform that we developed a series of numerical simulations and physical tests have been carried out. Results verify the superiority and effectiveness of our broken lines path following algorithm for unmanned surface vehicle especially in continuous broken lines following scenarios.
\end{abstract}

Keywords: Broken lines path following. disturbance uncertainties. unmanned surface vehicle. water experiment platform.

\section{Introduction}

With the aid of remote communication and automatic control technologies, unmanned surface vehicle (USV) excels in the flexible maneuverability, excellent autonomy 5 and concealment performance at sea, and can be conveniently loaded on other large vessels [1]. By employing 15 USV, an enormous amount of complex tasks [2, 3], including search and rescue, surveillance, reconnaissance, marine survey, and geological sampling can be independently and

\footnotetext{
* Corresponding author.

E-mail address: zhaoyujiaosl@126.com (Y. Zhao); qix-
} in.whut@gmail.com (X. Qi); Atilla.Incecik@strath.ac.uk (Atilla Incecik); myongdl@whut.edu.cn (Y. Ma); zhixiong_li@uow.edu.au (Z. $\mathrm{Li})$
10 successfully executed under rough sea conditions.

Several critical issues, including the path following, platform design contribute to the USV development. Referring to the path following problem, it plays a significant role in the intelligence and automation implementation of USV [4], which focuses on conceiving of an effective control scheme to guarantee USV sails along the preset paths. A path following control scheme consists of three parts, including path planning, path guidance, and velocity controller [5]. When it comes to the path planning problem, it is significant that a number of factors should be taken into account, including the depth, the traffic density, the obstacle-avoidance, the mainstream direction of the traffic flow. Accordingly, a sequence of way-points should be de- 
termined [6], by connecting continuous straight lines that 25 lowing problem, an USV should abide to the requirements of the given geometry paths, and motion boundary con- 65 straints. However, owing to the uncertainty disturbances brought by the time-varying environment $[7,8,9,10]$, including wind, wave, and current, it is extremely challenging for the USV to follow the planned paths. Meanwhile, to reduce the energy consumption, the transverse direction ${ }_{70}$ of the USV hull is not equipped with propulsion devices in general, and therefore there would not have sufficient control inputs. Hence by utilizing insufficient control inputs, the classical vector-based control method cannot directly resolve the USV path following problem. Consequently, it ${ }_{75}$ is necessary to devise a sound and logical path following scheme for the underactuated USV operating in an environment with random disturbances.

To resolve the USV path following or trajectory following problems, a number of excellent techniques have been ${ }^{80}$ proposed, including sliding mode control law [11], adaptive control approach [12], nonlinear guidance and fuzzy control [13], adaptive neural network control [14], and network-based controller [15]. However, the control methods in $[11,12,13,14]$ paid no attention to the random 85 environmental disturbances. For an underactuate USV, in [15] its motion model was simplified, and a fault detection filter and controller under network environments with actuator faults were designed. Referring to the surface vehicle path following problem where the disturbances due 90 to the random environment has been addressed, [4] utilized a dynamic model to contrive an adaptive control algorithm. [8] presented a novel trajectory tracking robust controller. [16] describes the formation control of underactuated USV by a neural networks-based dynamic surface 95 control approach. To compensate for drift forces through vehicle sideslip, [17] introduced a nonlinear adaptive pathfollowing controller, and [18] proposed a stable adaptive neural network tracking controller. It indicates that, the path following control methods in $[4,8,16,17,18]$ handled the adverse effects brought by the uncertain disturbances from the perspective of numerical simulations, and their feasibility and effectiveness have not yet been tested by experimental investigations.

Experimental verification is essential for the practical use of the above advance control algorithms. The authors of [19] experimentally resolved the path following issues for an underatcuated USV by employing a sliding mode control law. The authors of [20] constructed an adaptive control strategy for the path following of a surface vehicle, and validated the effectiveness of their algorithm by a scaled model vehicle. The authors of [21] paid great attention to the path following for surface vehicles with a novel back-stepping nonlinear controller, and validated the effectiveness of the controller by carrying out experiments with a model vehicle in a tank. However, when it comes to the followed paths in the validation of their control approaches in $[19,20,21]$, it can be found that only the lines and/or circles were chosen. Whereas the paths for surface vehicles are a series of broken lines. Consequently, for surface vehicle navigation, the above path following controllers may not be appropriate for direct application.

Following this conclusion, to investigate the path following problems for a USV with environmental random disturbances, this paper adopts carrot chasing algorithms and implements a broken lines path following algorithm for USV where the effectiveness of our algorithm is validated by numerical simulations and field experiments. Referring to the experimental validation, we build a suit of remote control system for a model USV with dual water jet propulsion. The utilized USV achieves the lateral drive through the control of the nozzle angle [22]. Compared with the variable pitch propeller, the water jet propulsion device also owns excellent acceleration and braking performance. Furthermore, the USV with dual water jet propulsion is featured by remarkable manoeuverability, small turning radius benefited from the lateral force, shallow draft, and 
concise gears [23]. As stated in [24], the servo motor has been widely used due to its classical control method. Consequently, we tend to employ the dual water jet propelled USV equipped with the servo motor for the path following control experiment validation.

Some highlights of this paper are: (1) To overcome the limitation that the followed paths in $[19,20,21]$ are lines or circles, and to satisfy the navigation practical requirements, we propose a broken lines path following algorithm for an underactuated USV, and the USV can follow a series of broken lines by employing our controller. (2) The manoeuverability of USV and the characteristics of carrot chasing algorithm are considered comprehensively, and then resolves the USV path following problems. (3) A dual water-jet propelled USV is selected as the test model, and numerical simulation and experiment results validate the effectiveness of our path following controller.

The remainder of the paper is organized as follows. Section 2 formulates the USV motion model for the control of a water-jet propelled USV, and details the path following problems for the USV. Section 3 proposes our path following algorithm, and states the modules of the USV selected for experiments. Section 4 validates the effectiveness of our controller with simulations and experiments. Section 5 gives conclusions.

\section{Problem Formulations}

\subsection{The water jet propulsion model of USV}

Fig. 1(a) shows the mechanical structure of a dual-jet propeller equipment on an underactuated USV, where $S_{e}$ is the servo motor, $o$ is the gravity center of the USV, and $o x$ and $o y$ are the longitudinal and transverse axes of the USV, respectively.

Referring to the navigation period, the USV has to adjust its motion state to overcome the adverse affects brought by the complex navigational condition [25]. As illustrated in Fig. 1(a), to generate the lateral driving force

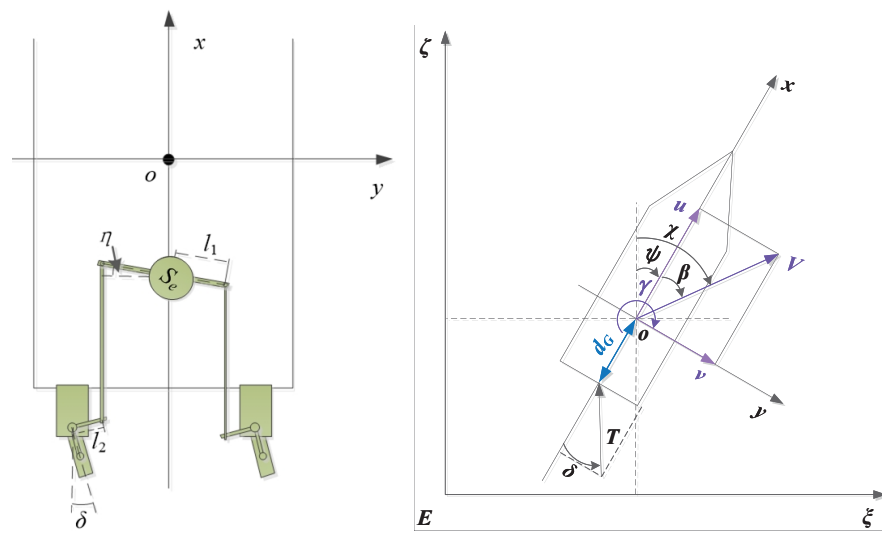

(a) A dual-jet propeller USV. (b) USV's coordination system.

Figure 1: Schematic of an underactuated USV.

for the USV, the angle of the jet nozzle $\delta$ should be controlled via the adjustment of the angle of the servo motor $\eta$. Where $\delta$ is the angle between the nozzle outlet and $o x$ of the USV, and is less than $90^{\circ}$. Suppose $l_{1}$ is the length of the connecting rod of the servo moto, and $l_{2}$ is the length of the oscillating head of the water jet nozzle. The relationship between $\delta$ and $\eta$ can be

$$
\delta=\arcsin \left(\frac{l_{1} \sin \eta}{l_{2}}\right), \quad \eta=\arcsin \left(\frac{l_{2} \sin \delta}{l_{1}}\right)
$$

Fig. 1(b) presents analysis of the force provided by the USV's jet propeller, where $T$ is the thrust generated by the jet propeller, $G\left(x_{G}, y_{G}\right)$ is the gravity center of the USV and coincides with $o, d_{G}$ is the distance from the thrust point of the jet to $G\left(x_{G}, y_{G}\right)$. Suppose $X_{c t r l}$ and $Y_{c t r l}$ denote the thrust on the $o x$ and oy directions of the USV, respectively. And $N_{c t r l}$ is the moment of-

fered by the jet propeller of the USV. Meanwhile, set $F_{c t r l}=\left[\begin{array}{lll}X_{c t r l} & Y_{c t r l} & N_{c t r l}\end{array}\right]^{T}$. Then,

$$
\left\{\begin{array}{l}
X_{c t r l}=T \cos \delta \\
Y_{c t r l}=-T \sin \delta \\
N_{c t r l}=T d_{G} \sin \delta
\end{array}\right.
$$

\subsection{Disturbance illustration}

During navigation, the USV is affected by the disturbances brought by the environments, including the wind, wave, and currents. Suppose $X, Y$, and $N$ are the surge 
force, the sway force, and the yaw moment generated by the disturbances, respectively. $X, Y$, and $N$ are the functions of the velocities $u, v$, and $\gamma$ and the corresponding accelerations $\dot{u}, \dot{v}$, and $\dot{\gamma}$, where $u$ and $v$ are the linear velocities in surge and sway, respectively, and $\gamma$ is the angular velocity in yaw. Consequently, the surge force $X_{\sum}$, the sway force $Y_{\sum}$, and the yaw moment $N_{\sum}$ on the USV can be $[26]$

$$
\left\{\begin{aligned}
X_{\Sigma}=X(u, v, \gamma, \dot{u}, \dot{v}, \dot{\gamma}) & +X_{c t r l} \\
= & X(u, v, \gamma, \dot{u}, \dot{v}, \dot{\gamma})+T \cos \delta \\
Y_{\sum}=Y(u, v, \gamma, \dot{u}, \dot{v}, \dot{\gamma}) & +Y_{c t r l} \\
= & Y(u, v, \gamma, \dot{u}, \dot{v}, \dot{\gamma})-T \sin \delta \\
N_{\sum}=N(u, v, \gamma, \dot{u}, \dot{v}, \dot{\gamma}) & +N_{c t r l} \\
= & N(u, v, \gamma, \dot{u}, \dot{v}, \dot{\gamma})+T d_{G} \sin \delta
\end{aligned}\right.
$$

Suppose $X_{*}, Y_{*}$, and $N_{*}$ are constant hydrodynamicderivative coefficients. When the disturbances are considered, details of $X, Y$, and $N$ can be

$$
\left\{\begin{aligned}
& X=X_{\dot{u}} \dot{u}+X_{u} u+X_{|u| u}|u| u+ X_{v \gamma} v \gamma \\
&+X_{\gamma \gamma} \gamma^{2}+\cdots \\
& Y=Y_{\dot{v}} \dot{v}+Y_{v} v+Y_{|v| v|v| v+Y_{\dot{\gamma}} \dot{\gamma}}+Y_{\gamma} \gamma \\
&\left.+Y_{|\gamma| \gamma}|\gamma| \gamma\right]+\cdots \\
& N=N_{\dot{v}} \dot{v}+N_{v} v+N_{|v| v}|v| v+N_{\dot{\gamma}} \dot{\gamma}+N_{\gamma} \gamma \\
&+N_{|\gamma| \gamma|\gamma| \gamma+\cdots}
\end{aligned}\right.
$$

To maintain the stability of the USV, its hull is symmetrically designed, and then $y_{G}=0$. Suppose $\boldsymbol{\nu}=\left[\begin{array}{lll}u & v & \gamma\end{array}\right]^{T}$, and $M=M_{R B}+M_{A}$. Wherein $M_{R B}$ and $M_{A}$ are the rigid mass matrix and the added mass matrix of the USV, respectively. Then, as formulated by Newton's Second Law, the force on the USV can be [27]

$$
M \dot{\nu}=F_{c t r l}+N(\boldsymbol{\nu}) \boldsymbol{\nu}
$$

where

$$
M_{R B}=\left[\begin{array}{ccc}
m & 0 & 0 \\
0 & m & 0 \\
0 & 0 & I_{Z Z}
\end{array}\right]
$$

$$
M_{A}=\left[\begin{array}{ccc}
-X_{\dot{u}} & 0 & 0 \\
0 & -Y_{\dot{v}} & m x_{G}-Y_{\dot{\gamma}} \\
0 & m x_{G}-N_{\dot{v}} & -N_{\dot{\gamma}}
\end{array}\right]
$$

and

$N(\boldsymbol{\nu})=\left[\begin{array}{ccc}X_{|u| u \mid}|u|+X_{u} & \left(m+X_{v \gamma}\right) \gamma & \left(m x_{G}+X_{\gamma \gamma}\right) \gamma \\ -m \gamma & Y_{|v| v}|v|+Y_{v} & Y_{|\gamma| \gamma} \gamma+Y_{\gamma} \\ -m x_{G} \gamma & N_{|v| v}|v|+N_{v} & N_{|\gamma| \gamma} \gamma+N_{\gamma}\end{array}\right]$

\subsection{Path following illustration}

To execute generous tasks, the underactuated USV should autonomously follow the preset paths as close as possible. However, owing to the disturbances stated in Section 2.2, trajectories of the USV are deviated from the preset paths through observation. Consequently, this paper focuses on the path following problem of the USV, and focuses on design an effective control strategy. By employing the control strategy, the USV would closely follow the preset paths within the allowed range of cross-track error.

Referring to the model of the USV, suppose the USV's motion can be represented by a three-freedom model in surge, sway, and yaw. As shown in Fig. 1(b), $\xi E \zeta$ is the earth coordinates, where $E \zeta$ and $E \xi$ point to North and East, respectively. And xoy is the USV's body fixed coordinates as stated in Fig. 1(a). Angle between $E \zeta$ and $o x$ is $\psi . V$ denotes the velocity of USV in $\xi E \zeta$, and its angles relate to $E \zeta$ and $o x$ are $\chi$ and $\beta$, respectively. $V_{\zeta}$ and $V_{\xi}$ are the axial components of $V$ in $E \zeta$ and $E \xi$, respectively. $\dot{V}, \dot{V}_{\zeta}$, and $\dot{V}_{\xi}$ correspond to the accelerations of $V, V_{\zeta}$, and $V_{\xi}$, respectively. $m$ and $I_{z z}$ denote the mass and the yaw moment of inertia of the USV in sequence. Consequently, the model of the USV can be [28]

$$
\left\{\begin{array}{l}
m\left(\dot{u}-y_{G} \dot{\gamma}-v \gamma-x_{G} \gamma^{2}\right)=X_{\Sigma} \\
m\left(\dot{v}+x_{G} \dot{\gamma}+u \gamma-y_{G} \gamma^{2}\right)=Y_{\sum} \\
I_{z z} \dot{\gamma}+m\left(x_{G}(\dot{v}+u \gamma)-y_{G}(\dot{u}-v \gamma)\right)=N_{\sum} \\
\dot{V}=\dot{u} \cos \beta+\dot{v} \sin \beta \\
\dot{V}_{\zeta}=\dot{V} \cos \gamma \\
\dot{V}_{\xi}=\dot{V} \sin \gamma
\end{array}\right.
$$


Referring to $T$, suppose $\epsilon$ is the size of the nozzle, $n$ is the propeller speed, and $b_{1}, b_{2}, k_{1}$, and $k_{2}$ are constants, then according to [27], it can be

$$
T=b_{1}|n| n+b_{2}|n| u=k_{1} \epsilon^{2 / 3}+k_{2} \epsilon^{1 / 3}
$$

In the earth coordination system $\xi E \zeta$, suppose $F_{\zeta c t r l}$ and $F_{\xi c t r l}$ are the forces provided by the water-jet propeller ${ }^{20}$ on the $E \zeta$ and $E \xi$, respectively, and $F_{N c t r l}$ is the steering force. Then, combined with Eq. (3), the above forces on the USV can be

$$
\left\{\begin{array}{l}
F_{\zeta c t r l}=X_{c t r l} \cos \psi-Y_{c t r l} \sin \psi \\
F_{\xi c t r l}=X_{c t r l} \sin \psi+Y_{c t r l} \cos \psi \\
F_{N c t r l}=N_{c t r l} \\
F_{\zeta c t r l}=X(u, v, \gamma, \dot{u}, \dot{v}, \dot{\gamma}) \cos \psi-Y(u, v, \gamma, \dot{u}, \dot{v}, \dot{\gamma}) \sin \psi \\
F_{\xi c t r l}=X(u, v, \gamma, \dot{u}, \dot{v}, \dot{\gamma}) \sin \psi+Y(u, v, \gamma, \dot{u}, \dot{v}, \dot{\gamma}) \cos \psi \\
F_{N c t r l}=N(u, v, \gamma, \dot{u}, \dot{v}, \dot{\gamma})
\end{array}\right.
$$

Similar to Eq. (3), in $\xi E \zeta$, referring to the forces on the USV on the $E \zeta$ and $E \xi$ are $F_{\zeta \sum}$ and $F_{\xi \sum}$, respectively, ${ }^{210}$ and $F_{N \sum}$ is the steering force on the USV. Then,

$$
\left\{\begin{array}{c}
F_{\zeta \sum}=F_{\zeta}+F_{\zeta c t r l} \\
F_{\xi \sum}=F_{\xi}+F_{\xi c t r l} \\
F_{N \sum}=F_{N}+F_{N c t r l}
\end{array}\right.
$$

Assuming that, the angle between the disturbance force on the USV and $o x$ is $\theta_{x}$ and equals to $\arctan \frac{F_{\zeta}}{F_{\xi}}+\frac{\pi}{2}-\psi$, where $\psi$ is the current course of the USV. If only the transverse disturbance force on the USV, then in $\xi E \zeta$, the force on oy can be $F_{\xi \Sigma}=\sqrt{F_{\zeta}^{2}+F_{\xi}^{2}} \sin \theta_{x}$ and $F_{\xi \Sigma}+T \sin \psi=0.220$ Consequently, the lateral disturbance force on the USV can
Meanwhile, set $l_{1}$ and $l_{2}$ are the length of the two sides that perpendicular to the spin axis of the USV. Then, the moment of inertia and torque of the USV can be

$$
\left\{\begin{array}{l}
I_{z z}=\frac{1}{12} m\left(l_{1}^{2}+l_{2}^{2}\right) \\
M_{N \sum}=F_{N \sum} d_{G}
\end{array}\right.
$$

Thus, assuming $\theta_{l}=\arcsin \left(\frac{l_{1} \sin \eta}{l_{2}}\right)$, then the details of
$F_{c t r l}$ of Eq. (8) can be defined as

$$
F_{c t r l}=\left[\begin{array}{c}
T \cos \theta_{l} \sin \psi+T \sin \theta_{l} \cos \psi \\
T \cos \theta_{l} \cos \psi-T \sin \theta_{l} \sin \psi \\
T d_{G} \sin \theta_{l}
\end{array}\right] .
$$

Consequently, the objective of the USV path following is that, by adjusting the water-jet propulsion speed $n$ and the motor's steering angle $\eta$, the cross-track errors would be converged to zero, and then the USV successfully follows the given paths.

\section{USV path following controller}

This section briefly states the straight line path following strategy, and proposes the path following algorithm for the USV that followed with a sequence of broken lines.

\subsection{Straight line path following strategy}

Fig. 2(a) shows the schematic of the straight line path following algorithm. In Fig. 2(a), suppose the line between points $W_{i}$ and $W_{i+1}$ is the preset path, $p(x, y)$ is the USV's current position, $q\left(x_{q}, y_{q}\right)$ is the projection of the point $p$ on the line $\overline{W_{i} W_{i+1}}, d_{e r}$ is the distance between USV's current position $p$ and USV's preset path $\overline{W_{i} W_{i+1}}$, and equals to the length of $\overline{p q}$, and $V, \psi$, and $\psi_{d}$ are the USV's current velocity, course angle, and heading angle, respectively. Meanwhile, $s\left(x_{t}, y_{t}\right)$ represents the virtual target point, the distance between $q$ and $s$ is $\lambda$, and the value of $\lambda$ can be varied with the requirements. $\theta$ represents the angle between $\overrightarrow{s p}$ and $\overrightarrow{s q}$. Set $\theta_{u}$ and $R_{u}$ are the angle and distance between $\overline{W_{i} p}$ and $\overline{W_{i} X}$, respectively. Set $\theta$ and $R$ are the angle and distance between $\overline{W_{i} q}$ and $\overline{W_{i} X}$, respectively. The geometric relationship in Fig. 2(a) can be

$$
\left\{\begin{array}{l}
R_{u}=\left\|W_{i}-p\right\| \\
\theta_{u}=\arctan \frac{y-y_{i}}{x-x_{i}} \\
\beta=\theta-\theta_{u} \\
R=R_{u} \cos \beta \\
d_{e r}=\sqrt{\left(x-x_{q}\right)^{2}+\left(y-y_{q}\right)^{2}}
\end{array}\right.
$$




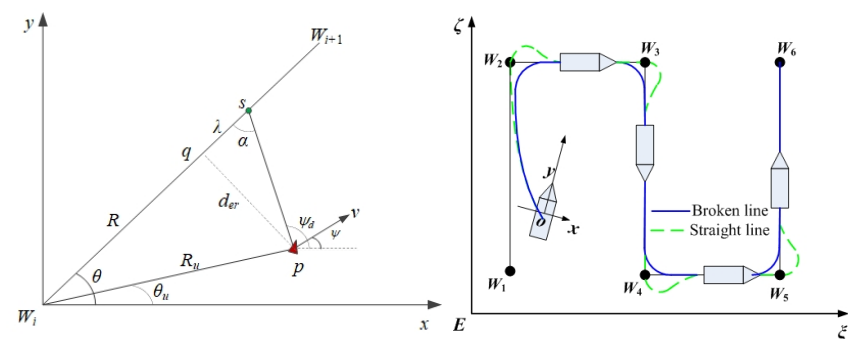

(a) Straight line path following (b) USV's path following stratealgorithm. gies.

Figure 2: Schematic of the straight line path following algorithm, and USV's path following strategies.

As stated in carrot chasing algorithm (CC) [29], the virtual target point (VTP) is introduced into the preset path, and the USV will follow the VTP. Referring to our straight line path following strategy for the USV, the dynamic VT$\mathrm{P} s\left(x_{t}, y_{t}\right)$ is added into $\overline{W_{i} W_{i+1}}$, and then the position of is calculated correspondingly. Following that, to proceed to $s$, the USV's $\psi$ and $V$ are adjusted by controlling its $n$ and $\eta$. And then, the position of $s\left(x_{t}, y_{t}\right)$ should be varied with the changing of $p(x, y)$, and the renewal method is listed in Eq. (13). Consequently, after iteration, the USV

According to Eq. (12), the virtual target point $s\left(x_{t}, y_{t}\right)$ and the desired heading angle $\psi_{d}$ of the USV can be

$$
\left\{\begin{array}{l}
x_{t}=(R+\lambda) \cos \theta \\
y_{t}=(R+\lambda) \sin \theta \\
\psi_{d}=\arctan \frac{y_{t}-y}{x_{t}-x}
\end{array}\right.
$$

Suppose $\Delta_{t}$ is the time interval, then the desired steering speed $\dot{\psi}_{d}$ can be

$$
\dot{\psi}_{d}=\frac{\psi_{d}-\psi}{\Delta_{t}}
$$

Meanwhile, $\ddot{\psi}_{d}$ can be derived from Eq. $(3,8,9,10)$ as below.

$$
\ddot{\psi}_{d}=\frac{N_{\sum}}{I_{z z}}
$$

Accordingly, $\ddot{\psi}_{d}$ can be

$$
\ddot{\psi}_{d}=12 d_{G} \frac{N+\left(k_{1} \epsilon^{2 / 3}+k_{2} \epsilon^{1 / 3}\right) d_{G} \sin \left(\arcsin \frac{l_{1} \sin \eta}{l_{2}}\right)}{m\left(l_{1}^{2}+l_{2}^{2}\right)}
$$

After the geometric analysis of Fig. 2(a), the following remarks are put forward for the USV linear path following problem.

Remark 1. The desired heading angle $\psi_{d}$ falls in $[0, \pi / 2]$ during the straight line path following period.

If $\theta_{u}<\theta, \psi_{d}=\theta+\arctan \frac{\left|d_{e r}\right|}{\lambda}$, where $\left|d_{e r}\right|$ and $\lambda$ are positive, and then $\arctan \frac{\left|d_{e r}\right|}{\lambda} \in[0, \pi / 2]$, and $\theta \leq \psi_{d} \leq \theta+\pi / 2$. Otherwise, when $\theta_{u} \geq \theta, \psi_{d}=\theta$-arctan $\frac{\left|d_{e r}\right|}{\lambda}$, and then $\theta-\pi / 2 \leq \psi_{d} \leq \theta$. Consequently, $\psi_{d} \in[0, \pi / 2]$.

Remark 2. The smaller the distance $d_{\text {er }}$ between the current position and the preset path of USV, the faster the velocity of advance of the USV that proceeds toward the preset path.

Suppose $v_{\|}$and $v_{\perp}$ denote the horizontal and vertical velocities along the direction the preset path, respectively, and the heading angle $\psi_{d}$ is kept with the course angle $\psi$,

If $\theta_{u}<\theta$, then $\psi=\theta+\arctan \frac{\left|d_{e r}\right|}{\lambda}$. According to Remark $1, \theta \leq \psi \leq \theta+\pi / 2$. Then $v_{\|}=v \cos (\psi-\theta)$ and $v_{\perp}=v \sin (\psi-\theta)$. Wherein $0 \leq \psi-\theta \leq \pi / 2$. Derived from Eq. (12), when $\left|d_{e r}\right|$ decreases, then $R_{u} \rightarrow R$, and $\theta_{u} \rightarrow \theta$. the value of $v_{\|}$is increases correspondingly.

If $\theta_{u} \geq \theta$, then $\psi=\theta$-arctan $\frac{\left|d_{e r}\right|}{\lambda}$. According to Remark $1, \theta-\pi / 2 \leq \psi \leq \theta$. Then $v_{\|}=v \cos (\theta-\psi)$ and $v_{\perp}=v \sin (\theta-\psi)$. Wherein $0 \leq \theta-\psi \leq \pi / 2$. Derived from Eq. (12), when $\left|d_{e r}\right|$ decreases, $\psi \rightarrow 0$, and then $\theta-\psi$ increases. Following that, $v_{\|}=v \cos (\theta-\psi)$ also increases.

Consequently, $v_{\|}$of the USV increases with the decreasing of $\left|d_{e r}\right|$. 


\subsection{Strategy for USV's broken lines path following}

During navigation practices, the USV generally follows the preset straight line path toward its destination. To avoid the non-navigable area, the preset path for the USV should be represented by a sequence of broken lines. Much attention has been paid to the straight line path following problem of USV, whereas little attention is paid to the broken lines path following problem [30]. It is evidently more practicable and generally that the USV should follow the broken lines. Following that, this section proposes a strategy for the USV's broken lines path following prob-

Fig. 2(b) shows the schematic of the path following strategies for the USV. Wherein, employing the straight line path following strategy and the broken lines path following strategy, the dashed green lines and the solid blue lines denote the corresponding following trajectories. It shows that at each straight line $\overline{W_{1} W_{2}}, \cdots, \overline{W_{5} W_{6}}$, to follow the above lines closely, the USV would proceed to the preset path continuously till arrived at the terminal points $W_{2}, \cdots, W_{6}$. It can be observed that, the USV deviates from its initial points $W_{1}, \cdots, W_{5}$ of the followed path visibly, and then the USV cannot follow the preset straight lines quite well. Consequently, to accommodate the broken lines path following issues for the USV, it is necessary to optimize the above straight line path following strategy.

As shown in Fig. 2(b), referring to the broken lines path following strategy for the USV, by calculating the position of the virtual target $s$ on each straight line $\overline{W_{1} W_{2}}$, $\cdots, \overline{W_{5} W_{6}}$, the USV can follow the virtual target dynamically. If the USV sails to the point $W_{2}$, and the target325 ken lines path following, we propose the broken lines path following algorithm (BLPFA) for the USV, and present the corresponding control procedure flow in Fig. 3. Firstly,

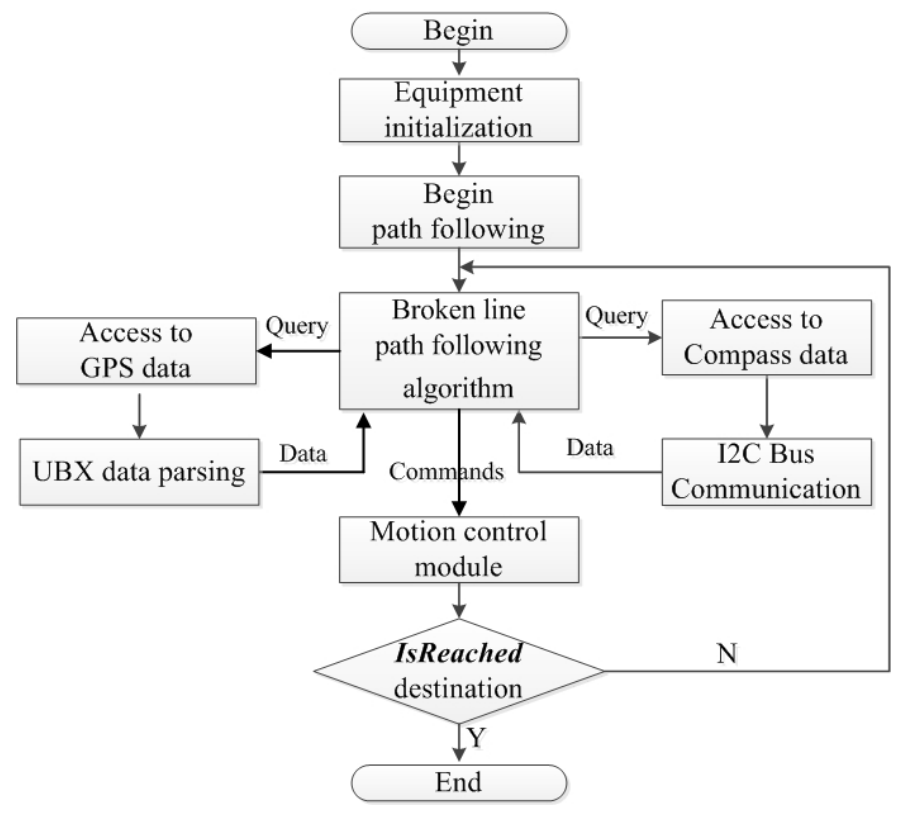

Figure 3: Procedure flow of the broken lines path following control for the USV.

waypoints information of the preset path is transmitted to the USV, and then the dynamic position $p(x, y)$ and course angle $\psi$ of the USV are accessed with the aid of GPS and geomagnetic device, respectively. Following that, by employing our BLPFA, the reached motion control commands Movecontrol are sent to the USV, and the USV follows the preset path smoothly. Finally, till the USV arrives at its destination with the assertion of IsReached, then the goal that the USV would follow the path represented by a sequence of broken lines, is achieved successfully.

\subsection{Implementation of the BLPFA on the USV}

Fig. 4 shows the composition diagram of the USV for BLPFA experimental validation. The USV consists of several modules, including data acquisition, data processing, and motion control modules. The data acquisition module can collect the position and course angle of the USV in real time. By utilizing the microprocessor, data processing module achieves the relationship between the preset path

When it comes to the details of the strategy for the bro- 


\author{
Algorithm $1 B L P F A$ \\ Input: \\ waypoint number $N_{w p}$ of the preset path; the $i$ th way- \\ point $W_{i}$ of the preset path, $i \leq N_{w p}$;

\section{Output:} \\ $F_{C o m}$ means the USV has followed all the preset path.
}

\section{Parameters:}

USV's current position $p(x, y)$ and course angle $\psi$, the virtual target $s\left(x_{t}, y_{t}\right)$, and the distance $\lambda$ between $q$ and $s$.

1: Initialize $i=1$.

2: while $i<N_{w p}-1$ do

3: for $W_{i} W_{i+1}$ do

4: $\quad$ Calculate $R, R_{u}, \theta, \theta_{u}, d_{e r}$ with Eq. (12);

5: $\quad$ Calculate $s\left(x_{t}, y_{t}\right), \psi_{d}$ with Eq. (13).

6: $\quad$ if $\overline{W_{i} s} \geq \overline{W_{i} W_{i+1}}$ then

7: $\quad$ Calculate $R^{\prime}, R_{u}^{\prime}, \theta^{\prime}, \theta_{u}^{\prime}, d_{e r}^{\prime}$ with Eq. (12) wherein $i=i+1$;$$
\text { if } d_{e r}<d_{e r}^{\prime} \text { then }
$$$$
s\left(x_{t}, y_{t}\right)=W_{i+1} \text {. }
$$

else

$$
s\left(x_{t}, y_{t}\right)=s^{\prime}\left(x_{t}, y_{t}\right) .
$$

end if

$i=i+1$.

Update $W_{i}, W_{i+1}, \lambda$;

\section{end if}

$$
\operatorname{Movecontrol}\left(\psi, \psi_{d}, p(x, y), s\left(x_{t}, y_{t}\right)\right) \text {. }
$$

\section{end for}

if IsReached $\left(p(x, y), W_{i}\right)$ then return $F_{C o m}$.

end if and the current position of the USV, and then offers the desired motion control commands for the USV following above control commands.

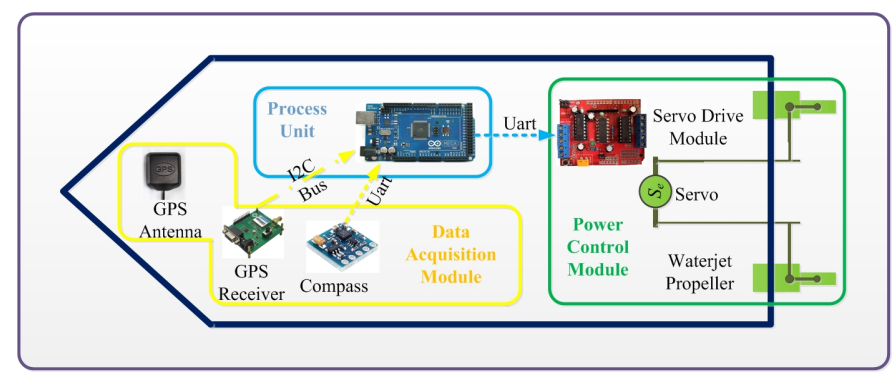

Figure 4: Composition diagram of the USV for BLPFA experimental vaildation.

The data acquisition module integrates the GPS module 'EPPINNO-A10', and the geomagnetic module 'QMC5883L'. Wherein the GPS module 'EPPINNO-A10' sup-

$$
\text { Calculate } s^{\prime}\left(x_{t}, y_{t}\right), \psi_{d} \text { with Eq. (13). }
$$
ports the frequency signal L1, including GPS C/A and SBAS, and is widely applied for generous OEM/ODM schemes. QMC5883L is a multi-chip triaxial magnetic sensor and suitable for the navigation with high-accuracy precision. The data processing module adopts Arduino Mega2560. The motion control module selects the BTS7960 driving module, and can drive 4 DC motors with the peak current $43 \mathrm{~A}$ per channel directly.

\section{Numerical simulation and experiments valida- tion}

To validate the effectiveness of our BLPFA for the USV, this section describes the simulations and water experiments carried out under various scenarios. Units of length, and velocity are in meter, and meter per second respectively. For each case, it has four figures in $\xi E \zeta$ system, including the paths, the distance error of the paths $d_{e r}$, the velocity $v$, and the course angle $\psi$ of the USV. The sampling number is $N_{\text {sam }}$, and $\psi$ ranges from $0^{\circ}$ to $360^{\circ}$, where $0^{\circ}$ and $360^{\circ}$ overlap each other. 


\subsection{Simulations}

To strengthen the reliability and dismiss the contingency 395 of our simulation work, as shown in Fig. 5, two typical preset paths denoted by black dashed lines, including a closed polygon path, and a circular path of [31] are utilized for the simulations. To validate the superiority of our BLPFA, two excellent algorithms including CC and ILOS+NN [31 ]400 are utilized for comparison. Within ILOS+NN algorithm, it consists of integral line-of-sight (LOS) guidance [32] and robust RBFNN compensations, and its goal of path following can be reached by controlling the movement of USV to decrease the along-error and cross-error. Suppose $T=3 \mathrm{~N}, 405$ $m=30 \mathrm{~kg}, \delta \in[-\pi / 3, \pi / 3]$, the time step $\Delta t=1 \mathrm{~s}, \lambda=1 \mathrm{~m}$ in low velocity cases and $\lambda=3 \mathrm{~m}$ in high velocity cases, and the time-varying environmental disturbances can be

$$
\left\{\begin{array}{l}
F_{\zeta}=0.2+0.3 \sin 0.02 t+0.2 \sin 0.08 t+0.3 \text { rand } \\
F_{\xi}=-0.15+0.4 \cos 0.01 t+0.25 \sin 0.05 t+0.2 \text { rand }_{410} \\
F_{N}=-0.5 \sin 0.09 t-0.3 \sin 0.01 t+0.2 \text { rand }
\end{array}\right.
$$

Referring to USV's path following simulation with the

\section{$\mathrm{ILOS}+\mathrm{NN}$}

Following that, corresponding curves of the velocities $v$ and the course angle $\psi$ adopted in our BLPFA, CC and ${ }_{425}$ ILOS $+\mathrm{NN}$ are listed in Fig. 9 and Fig. 10, respectively. each other, then if $\psi_{A}$ and $\psi_{B}$ are around $0^{\circ}$ and $360^{\circ}$, and then the variation between $\psi_{A}$ and $\psi_{B}$ is quite small, due to they are close to each other. Therefore, it can be 430

concluded that the course angle $\psi$ in Fig. 10 is relatively smooth, $\psi$ is disturbed when there exist disturbances, and the convergence of the curves of $\psi$ achieved by our BLPFA is faster than that of the other two algorithms. All the phenomena validate the effectiveness of our BLPFA.

With the aid of our BLPFA, benefited from the dynamic adjustable of the water jet propulsion USV's control parameters, including $n$ and $\eta$, and then the USV's course can be modified properly. Following that, our strategy is featured by smaller $d_{e r}$ and minor amplitude variations of $\psi$. Consequently, the path following effects with our strategy is better than those of the other two algorithms. Furthermore, our BLPFA can resolve the USV's path following issues with both broken lines and circles, and then compared with $\mathrm{CC}$ and ILOS+NN its compatibility is stronger.

Remark 3. It can be observed that, the simulations reveal the superiority of our BLPFA than $C C$ and $I L O S+N N$ under uncertain adverse environmental disturbances. Constrained by environmental constraints, it is impossible to carry out water tests under high sea conditions. Following that, the experiments were carried out under general wind and wave conditions.

\subsection{Water experiments}

As shown in Fig. 11(a), water experiments were carried out in a rectangular towing tank with a size of $75 \mathrm{~m} \times 55 \mathrm{~m}$ located in Wuhan University of Technology, China. Environmental disturbances are mainly brought by breeze and small waves. A continuous $\mathrm{V}$-shaped path with ten waypoints $w_{p i}(i=1, \ldots, 10)$ listed in Table 1 is set for the USV. The scenario that the USV follows the preset path is given in Fig. 11(b). The USV is with a size of $1.80 \mathrm{~m} \times 0.30 \mathrm{~m}$ and a weight of $20 \mathrm{~kg}$, and its velocity $V=1.5 \mathrm{~m} / \mathrm{s}$.

Fig. 11(b) shows the followed path and the preset Vshaped paths denoted by dotted yellow lines, and solid white lines, respectively. Resulting from the uncertain disturbances, there exist $d_{e r}$ and course angle differences $\Delta \psi$ 


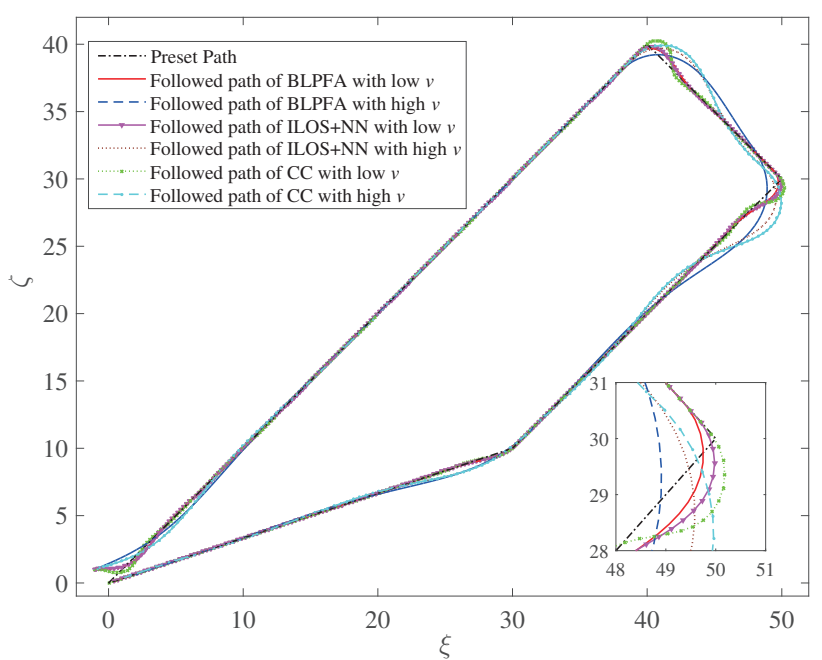

(a) Broken lines path following.

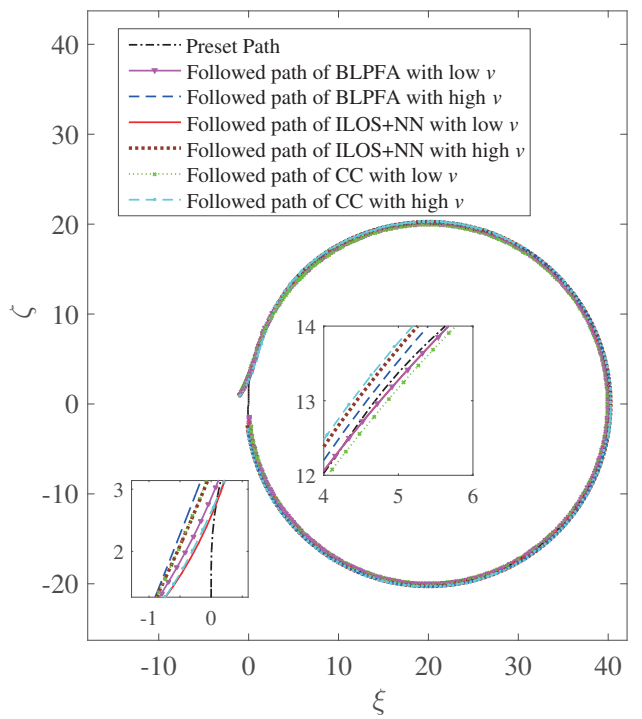

(b) Circular path following.

Figure 5: Following two preset paths without disturbance and with different velocities by employing BLPFA, CC and ILOS+NN.

Table 1: Positions of the preset paths

\begin{tabular}{lccccc}
\hline$W_{p i}$ & latitude & longitude & $W_{p i}$ & latitude & longitude \\
\hline$W_{p 1} 30^{\circ} 36^{\prime} 34.54^{\prime \prime}$ & $114^{\circ} 21^{\prime} 15.54^{\prime \prime}$ & $W_{p 6}$ & $30^{\circ} 36^{\prime} 33.10^{\prime \prime}$ & $114^{\circ} 21^{\prime} 17.51^{\prime \prime}$ \\
$W_{p 2}$ & $30^{\circ} 36^{\prime} 32.92^{\prime \prime}$ & $114^{\circ} 21^{\prime} 16.39^{\prime \prime}$ & $W_{p 7}$ & $30^{\circ} 36^{\prime} 34.91^{\prime \prime}$ & $114^{\circ} 21^{\prime} 17.27^{\prime \prime}$ \\
$W_{p 3}$ & $30^{\circ} 36^{\prime} 34.62^{\prime \prime}$ & $114^{\circ} 21^{\prime} 16.12^{\prime \prime}$ & $W_{p 8}$ & $30^{\circ} 36^{\prime} 33.25^{\prime \prime}$ & $114^{\circ} 21^{\prime} 18.08^{\prime \prime}$ \\
$W_{p 4}$ & $30^{\circ} 36^{\prime} 32.98^{\prime \prime}$ & $114^{\circ} 21^{\prime} 16.95^{\prime \prime}$ & $W_{p 9}$ & $30^{\circ} 36^{\prime} 35.07^{\prime \prime}$ & $114^{\circ} 21^{\prime} 17.88^{\prime \prime}$ \\
$W_{p 5} 30^{\circ} 36^{\prime} 34.76^{\prime \prime}$ & $114^{\circ} 21^{\prime} 16.70^{\prime \prime}$ & $W_{p 10}$ & $30^{\circ} 36^{\prime} 33.36^{\prime \prime}$ & $114^{\circ} 21^{\prime} 18.69^{\prime \prime}$ \\
\hline
\end{tabular}

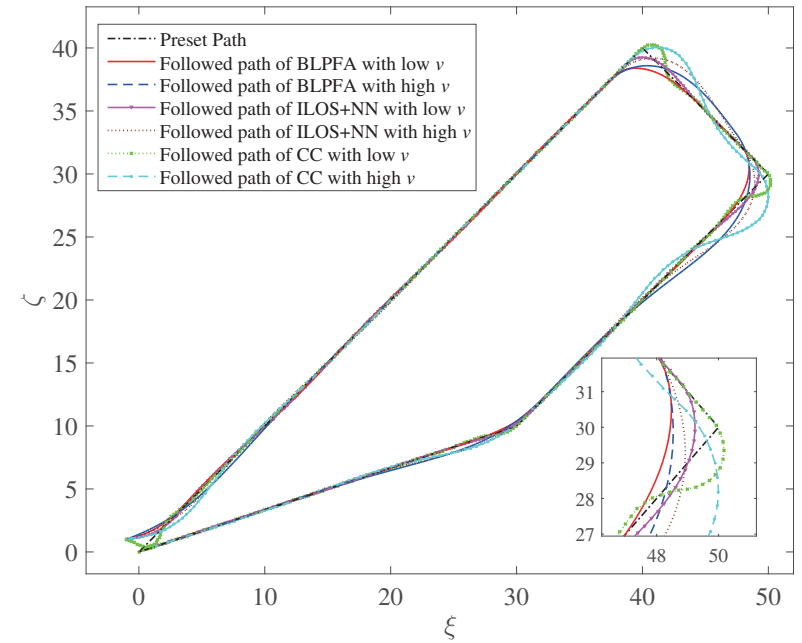

(a) Broken lines path following.

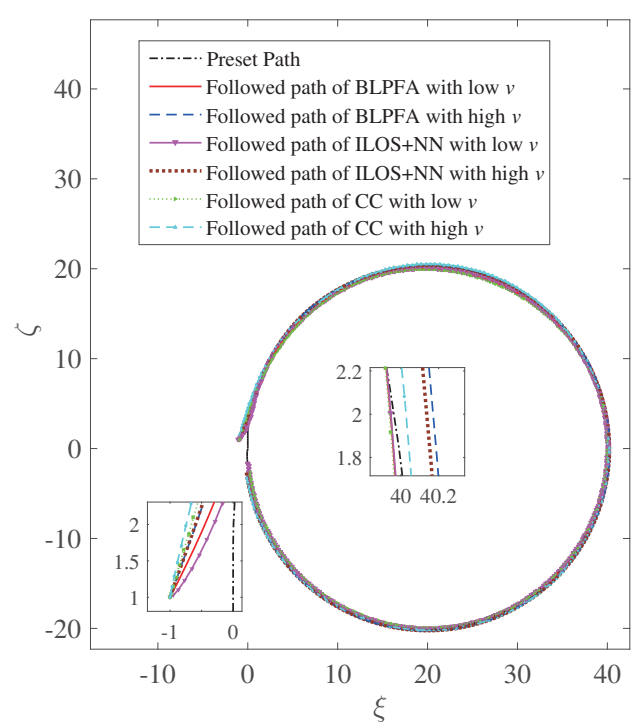

(b) Circular path following.

Figure 6: Following two preset paths with disturbances and different velocities by employing BLPFA, CC and ILOS+NN . 


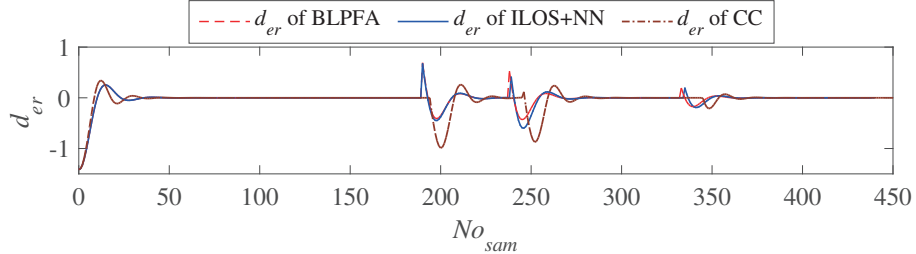

(a) $d_{e r}$ with low velocity, without disturbance.

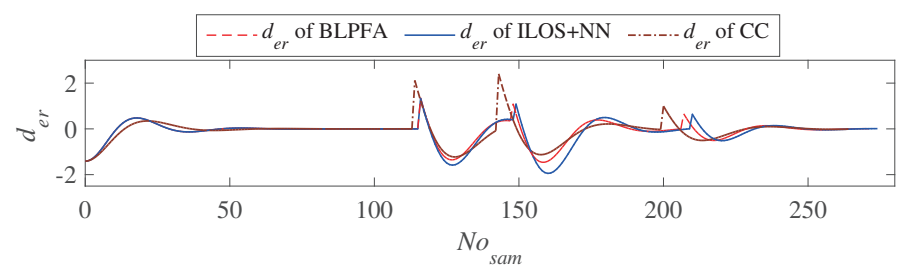

(b) $d_{e r}$ with high velocity, without disturbance.

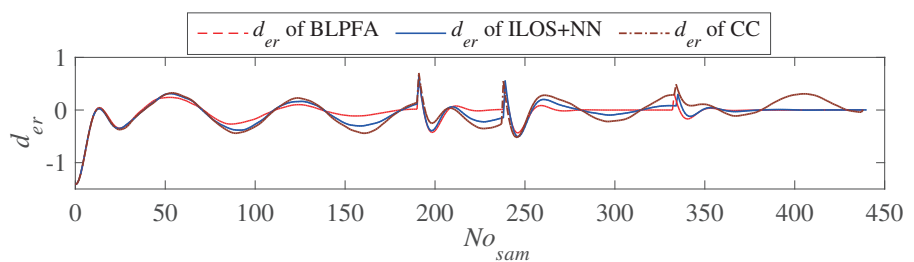

(c) $d_{e r}$ with low velocity, with disturbance.

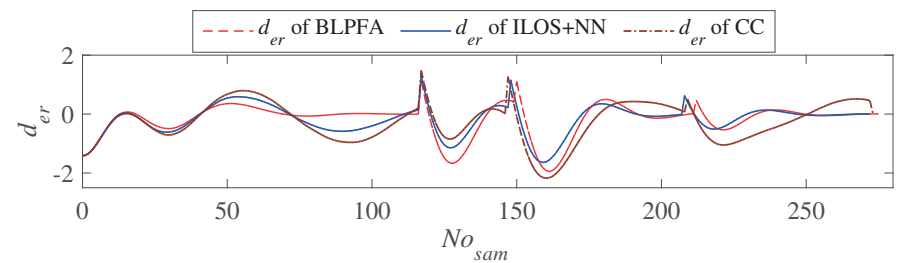

(d) $d_{e r}$ with high velocity, with disturbance.

Figure 7: $d_{e r}$ of the broken lines cases in Fig. 5(a) and Fig. 6(a) by employing BLPFA, CC and ILOS+NN.

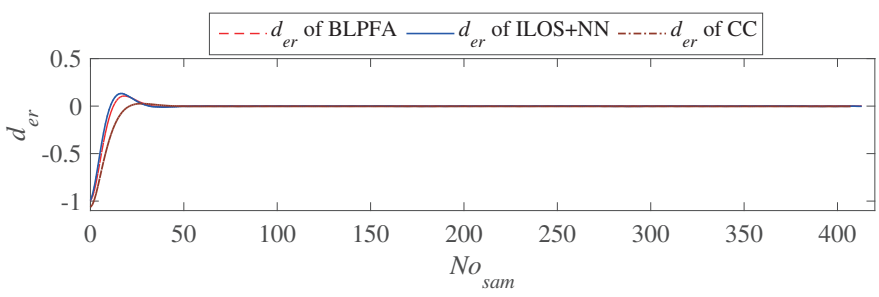

(a) $d_{e r}$ with low velocity, without disturbance.

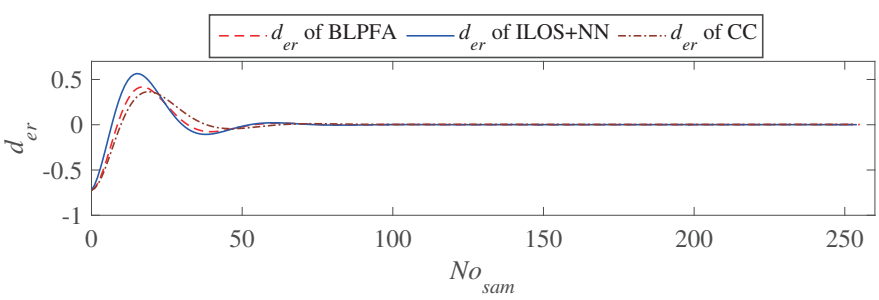

(b) $d_{e r}$ with high velocity, without disturbance.

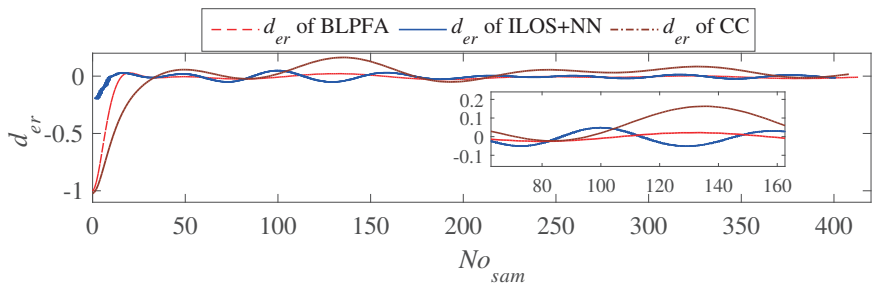

(c) $d_{e r}$ with low velocity, with disturbance.

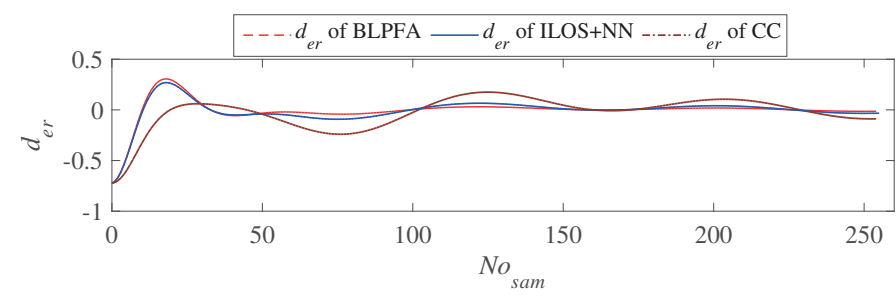

(d) $d_{e r}$ with high velocity, with disturbance.

Figure 8: $d_{e r}$ of the circular cases in Fig. 5(b) and Fig. 6(b) by employing BLPFA, CC and ILOS+NN.
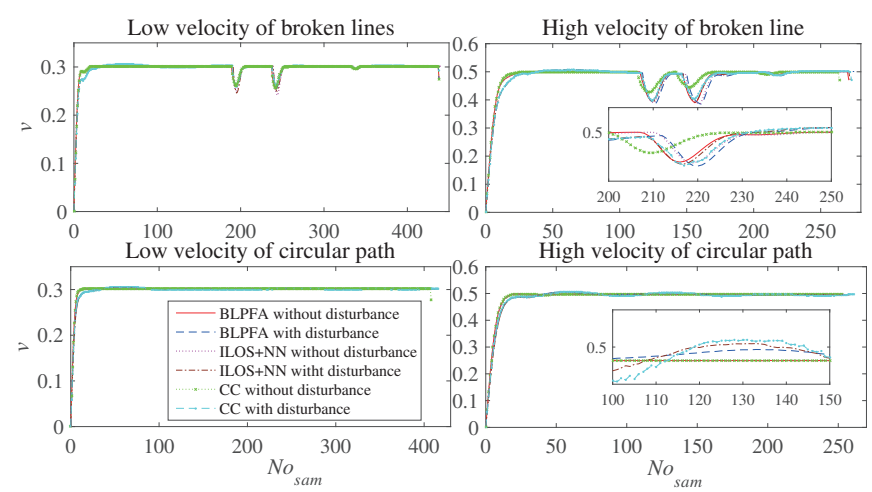

Figure 9: $v$ of the two preset paths for following by employing BLPFA, CC and ILOS+NN. 

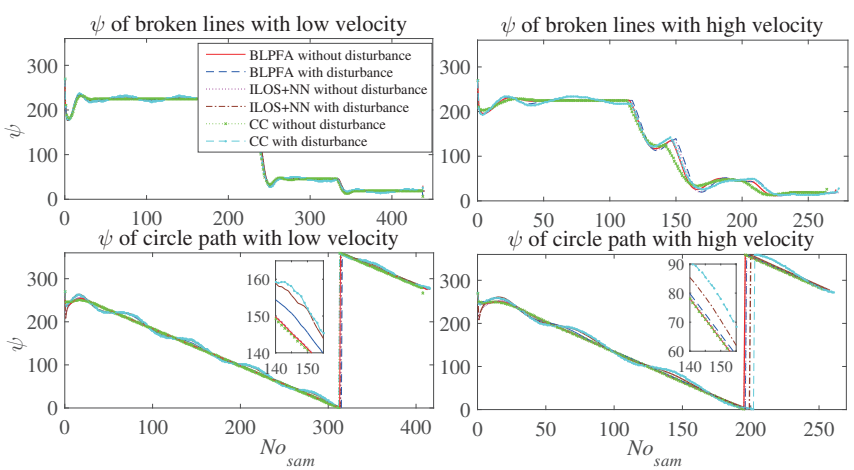

Figure 10: $\psi$ of the two preset paths for following by employing BLPFA, CC and ILOS+NN.

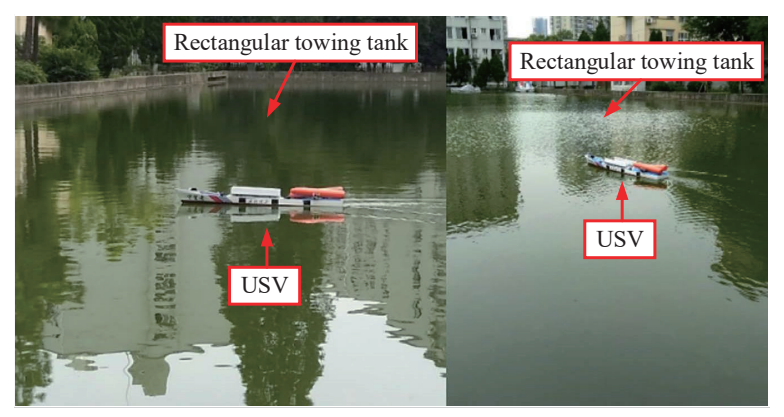

(a) USV employed for experiments.

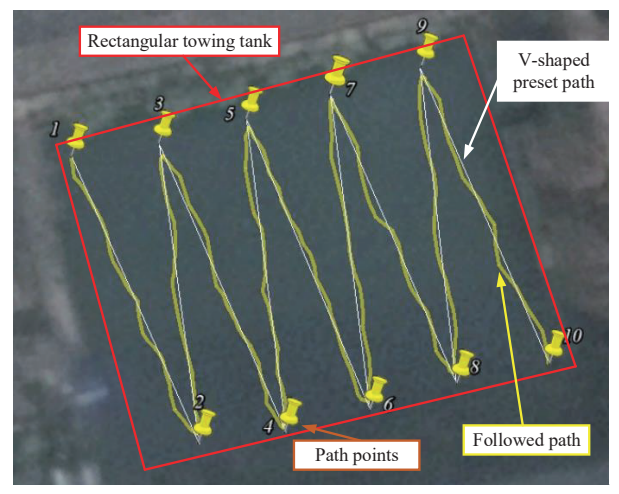

(b) USV's preset and followed paths.

Figure 11: Experiment scenarios and employed USV.

between the above two paths as shown in Fig. 12. The value of $d_{e r}$ is generally less than $1 \mathrm{~m}$, except around the waypoints is also less than $1.3 \mathrm{~m}$. The range of $\Delta \psi$ is $\mathrm{kept}_{455}$ in $\left[-20^{\circ}, 20^{\circ}\right]$, expect around the waypoints which is also less than $\pm 50^{\circ}$. However, benefited from our BLPFA, as shown in Fig. 11(b) and Fig. 12, the USV dynamically adjusts its motion state, maintains $d_{e r}$ and $\Delta \psi$ around a small value, and finally proceeds to the destination suc- 460 cessfully.
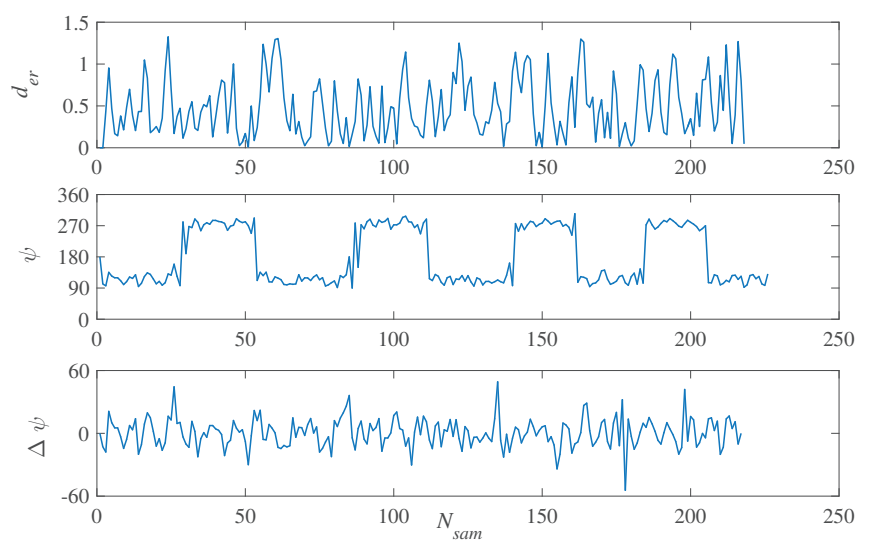

Figure 12: Results of the path following experiments of the USV, including $d_{e r}, \psi$, and $\Delta \psi \cdot d_{e r}$ : the distance between USV's current position $p$ and USV's preset path $\overline{W_{i} W_{i+1}}, \psi$ : the heading angle, $\Delta \psi$ : angle of heading to be changed.

Consequently, resulting from the excellent performance of our BLPFA in the water experiments, it can be validated that, our proposed control strategy for the USV's broken lines path following is effective.

\section{Conclusions} BLPFA guarantees that the USV follows the preset path smoothly and closely with a small path difference. The numerical simulations and water experiments verify the effectiveness of our broken lines path following algorithm. In the near future, combining with the guidance systems, we would consider the integration of guidance, navigation and intelligent control algorithm on this platform to realize the intellectualization of USV, and improve its path following performance. Then we would put our path following controller to use for large-scale USVs. 


\section{Acknowledgments}

The authors sincerely thank the Editor-in-Chief, associate Editor and anonymous reviewers for their insightful, constructive and valuable comments which lead to the better presentation of this paper. The authors are partially supported by the National Science Foundation of China (51579202, 51911540478, 61673223 and 51309186), China Postdoctoral Science Foundation Funded Project (2015T80848 and 2014M560633), and National key R \& D plan (2018YFC1407400).

\section{References}

[1] H. Niu, Y. Lu, A. Savvaris, A. Tsourdos, An energy-efficient path planning algorithm for unmanned surface vehicles, Ocean

[3] G. Zhang, Y. Deng, W. Zhang, Robust neural path-following control for underactuated ships with the dvs obstacles avoidance guidance, Ocean Engineering 143 (2017) 198-208.

[4] J. Shin, D. J. Kwak, Y.-i. Lee, Adaptive path-following control for an unmanned surface vessel using an identified dynamic model, IEEE/ASME Transactions on Mechatronics 22 (3) (2017) 1143-1153.

[5] H. Katayama, H. Aoki, Straight-line trajectory tracking control ${ }^{535}$ for sampled-data underactuated ships, IEEE Transactions on Control Systems Technology 22 (4) (2014) 1638-1645.

[6] Y. Ma, M. Hu, X. Yan, Multi-objective path planning for unmanned surface vehicle with currents effects, ISA transactions 75 (2018) 137-156.

[7] N. Wang, S. Lv, W. Zhang, Z. Liu, M. J. Er, Finite-time observer based accurate tracking control of a marine vehicle with complex unknowns, Ocean Engineering 145 (2017) 406-415.

[8] Y. Yang, J. Du, H. Liu, C. Guo, A. Abraham, A trajectory tracking robust controller of surface vessels with disturbance ${ }^{545}$ uncertainties, IEEE Transactions on Control Systems Technology 22 (4) (2014) 1511-1518.

[9] L. Liu, D. Wang, Z. Peng, Path following of marine surface vehicles with dynamical uncertainty and time-varying ocean disturbances, Neurocomputing 173 (2016) 799-808.

[10] H. Kim, S.-H. Kim, M. Jeon, J. Kim, S. Song, K.-J. Paik, A study on path optimization method of an unmanned surface ve- hicle under environmental loads using genetic algorithm, Ocean Engineering 142 (2017) 616-624.

[11] Z. Sun, G. Zhang, B. Yi, W. Zhang, Practical proportional integral sliding mode control for underactuated surface ships in the fields of marine practice, Ocean Engineering 142 (2017) 217223.

[12] Y.-Y. Chen, Y.-P. Tian, Coordinated adaptive control for threedimensional formation tracking with a time-varying orbital velocity, IET Control Theory \& Applications 7 (5) (2013) 646.

[13] C. Yu, X. Xiang, L. Lapierre, Q. Zhang, Nonlinear guidance and fuzzy control for three-dimensional path following of an underactuated autonomous underwater vehicle, Ocean Engineering 146 (2017) 457-467.

[14] S.-L. Dai, M. Wang, C. Wang, Neural learning control of marine surface vessels with guaranteed transient tracking performance, IEEE Transactions on Industrial Electronics 63 (3) (2016) 17171727.

[15] Y.-L. Wang, Q.-L. Han, Network-based fault detection filter and controller coordinated design for unmanned surface vehicles in network environments, IEEE Transactions on Industrial Informatics 12 (5) (2016) 1753-1765.

[16] Z. Peng, D. Wang, Z. Chen, X. Hu, W. Lan, Adaptive dynamic surface control for formations of autonomous surface vehicles with uncertain dynamics, IEEE Transactions on Control Systems Technology 21 (2) (2013) 513-520.

[17] T. I. Fossen, K. Y. Pettersen, R. Galeazzi, et al., Line-of-sight path following for dubins paths with adaptive sideslip compensation of drift forces., IEEE Trans. Contr. Sys. Techn. 23 (2) (2015) 820-827.

[18] S.-L. Dai, C. Wang, F. Luo, Identification and learning control of ocean surface ship using neural networks, IEEE Transactions on Industrial Informatics 8 (4) (2012) 801-810.

[19] H. Ashrafiuon, K. R. Muske, L. C. McNinch, R. A. Soltan, Sliding-mode tracking control of surface vessels, IEEE transactions on industrial electronics 55 (11) (2008) 4004-4012.

[20] R. Skjetne, T. I. Fossen, P. V. Kokotović, Adaptive maneuvering, with experiments, for a model ship in a marine control laboratory, Automatica 41 (2) (2005) 289-298.

[21] Z. Li, J. Sun, S. Oh, Design, analysis and experimental validation of a robust nonlinear path following controller for marine surface vessels, Automatica 45 (7) (2009) 1649-1658.

[22] D. Wu, F. Ren, W. Zhang, An energy optimal thrust allocation method for the marine dynamic positioning system based on adaptive hybrid artificial bee colony algorithm, Ocean Engineering 118 (C) (2016) 216-226.

[23] T. Takai, M. Kandasamy, F. Stern, Verification and validation study of urans simulations for an axial waterjet propelled large high-speed ship, Journal of Marine Science and Technolo- 
gy 16 (4) (2011) 434-447.

[24] Y. Wang, Y. Zhao, S. A. Bortoff, K. Ueda, A real-time energyoptimal trajectory generation method for a servomotor system, IEEE Transactions on Industrial Electronics 62 (2) (2015) 11751188.

[25] Z. Peng, D. Wang, W. Wang, L. Liu, Neural adaptive steering of an unmanned surface vehicle with measurement noises, Neurocomputing 186 (2016) 228-234.

[26] C. Sonnenburg, A. Gadre, D. Horner, S. Kragelund, A. Marcus, D. J. Stilwell, C. Woolsey, Control-oriented planar motion modeling of unmanned surface vehicles, in: OCEANS $2010 \mathrm{MT}-$ S/IEEE SEATTLE, IEEE, 2010, pp. 1-10.

565 [27] J. Han, J. Xiong, Y. He, F. Gu, D. Li, Nonlinear modeling for a water-jet propulsion usv: An experimental study, IEEE Transactions on Industrial Electronics 64 (4) (2017) 3348-3358.

[28] T. I. Fossen, Handbook of marine craft hydrodynamics and motion control, John Wiley \& Sons, 2011.

[29] P. Sujit, S. Saripalli, J. B. Sousa, Unmanned aerial vehicle path following: A survey and analysis of algorithms for fixed-wing unmanned aerial vehicless, IEEE Control Systems 34 (1) (2014) $42-59$.

[30] H. Kim, B. K. Kim, Online minimum-energy trajectory planning and control on a straight-line path for three-wheeled omnidirectional mobile robots., IEEE Trans. Industrial Electronics 61 (9) (2014) 4771-4779.

[31] Z. Zheng, L. Sun, Path following control for marine surface vessel with uncertainties and input saturation, Neurocomputing 177 (2016) 158-167.

[32] L. Liu, D. Wang, Z. Peng, H. Wang, Predictor-based los guidance law for path following of underactuated marine surface vehicles with sideslip compensation, Ocean Engineering 124 (2016) 340-348. 